\title{
Spectrum Sharing and Sensing for Future Broadband Networks: The Cognitive Radio Technology
}

\author{
Massimiliano Laddomada, ${ }^{1}$ Hsiao Hwa Chen, ${ }^{2}$ Fred Daneshgaran, ${ }^{3}$ Marina Mondin, ${ }^{4}$ \\ and Hamid Sadjadpour ${ }^{5}$ \\ ${ }^{1}$ Department of Electrical Engineering, Texas A\&M University, Texarkana, TX 75505, USA \\ ${ }^{2}$ National Cheng Kung University, Taiwan \\ ${ }^{3}$ California State University, Los Angeles, USA \\ ${ }^{4}$ Politecnico di Torino, Italy \\ ${ }^{5}$ University of California, Santa Cruz, USA
}

Correspondence should be addressed to Massimiliano Laddomada, mladdomada@tamut.edu

Received 26 June 2010; Accepted 29 June 2010

Copyright (C) 2010 Massimiliano Laddomada et al. This is an open access article distributed under the Creative Commons Attribution License, which permits unrestricted use, distribution, and reproduction in any medium, provided the original work is properly cited.

A recent measurement campaign on spectrum utilization promoted by the Federal Communication Commission throughout the US has revealed that significant portions of the electromagnetic spectrum are rarely exploited for considerable intervals of time, thus paving the way to a number of possibilities for increasing bandwidth utilization among multiple users in shared radio networks. The clue to improve spectrum utilization, as well as efficiency, comes from communications systems exploiting the socalled Cognitive Radio technology, which at the physical layer, as well as at upper-levels of the network, can adaptively and dynamically allow users to access radio resources by switching among portions of unused bandwidth in different intervals of time. The cognitive radio technology is still in its infancy, and many problems at a theoretical as well as practical level have to be solved before this technology may be fully exploited in next generation wireless networks.

This special issue is aimed at highlighting state-ofthe-art techniques on the most recent research advances in cognitive radio networks. The first paper presents an optimization framework for collaborative spectrum sensing in terms of optimum decision fusion for hard and soft decision combining. One of the main conclusions of the work is that, for optimum fusion, the fusion centre must incorporate signal-to-noise ratio values of cognitive users and the channel conditions. A genetic algorithm based on weighted optimization strategy is also presented for the case of soft decision combining. In the second paper, a fast detection scheme consisting of multiple cognitive radios and a central control office is proposed. Specifically, each cognitive radio makes individual detection decision using the sequential probability ratio test combined with Neyman Pearson detection with respect to a specific observation window length. The third paper proposes the establishment of a simultaneous cognitive radio communication based on a subdistribution of power made over unselected subchannels that were discarded by the primary user through an initial optimal power allotment. The aim of the work is to show the possibility of introducing an opportunistic communication into a licensed transmission where the total power constraint is shared. The fourth paper proposes an algorithm for cooperative Dynamic Spectrum Access in Cognitive Radio networks utilizing Medium Access Control layer mechanisms for message exchange between secondary nodes in order to achieve interference mitigation. The proposed algorithm is applied in Filter Bank Multicarrier and Orthogonal Frequency Division Multiplexing systems. The fifth paper proposes an optimization technique based on genetic algorithms for optimal collaborative multiband sensing. Since Genetic programming performs a direct search of the optimal solution without approximations and solution domain restrictions, collaborative multiband sensing can be consistently optimized without limitations. The sixth paper investigates the application of a parallel compressive sensing 
architecture for wideband spectrum sensing at sub-Nyquist rates by exploiting the current reduced frequency usage. The proposed architecture possesses attractive spurious frequency rejection schemes that are critical in the implementation of spectrum sensing systems. The seventh paper describes a cross-layer throughput optimization problem based on a modified signal-to-interference-plus-noise ratio. The objective is to maximize the minimum end-to-end flow throughput. The eighth paper presents a review of the state of the art in technology, regulation, and standardization of cognitive access to TV White Spaces. It examines the spectrum opportunity and commercial use cases associated with this form of secondary access. The ninth paper strives to provide a solution to the hidden-node problem for passive-listening receiver based on cooperation of multiple cognitive radios. The work considers a cooperative GPSenabled cognitive network. Once the presence of primary users is detected, a localization algorithm is employed to first estimate the path loss model for the environment based on back propagation method and then to locate the position of primary user. In the tenth paper, two complementary algorithms based on information theoretic measures of statistical distribution divergence and information content are proposed. The first method is applicable to signals with periodic structures and is based on the analysis of KullbackLeibler divergence. The second exploits information content analysis to detect unusual events. The last paper of this special issue focuses on a frequency-designed fractional delay FIR structure suitable for software radio applications. The design method is based on frequency optimization of a combination of modified Farrow and multirate structures.

The authors are grateful to the reviewers for their invaluable work and to the authors of the papers collected in this special issue.

\author{
Massimiliano Laddomada \\ Hsiao Hwa Chen \\ Fred Daneshgaran \\ Marina Mondin \\ Hamid Sadjadpour
}



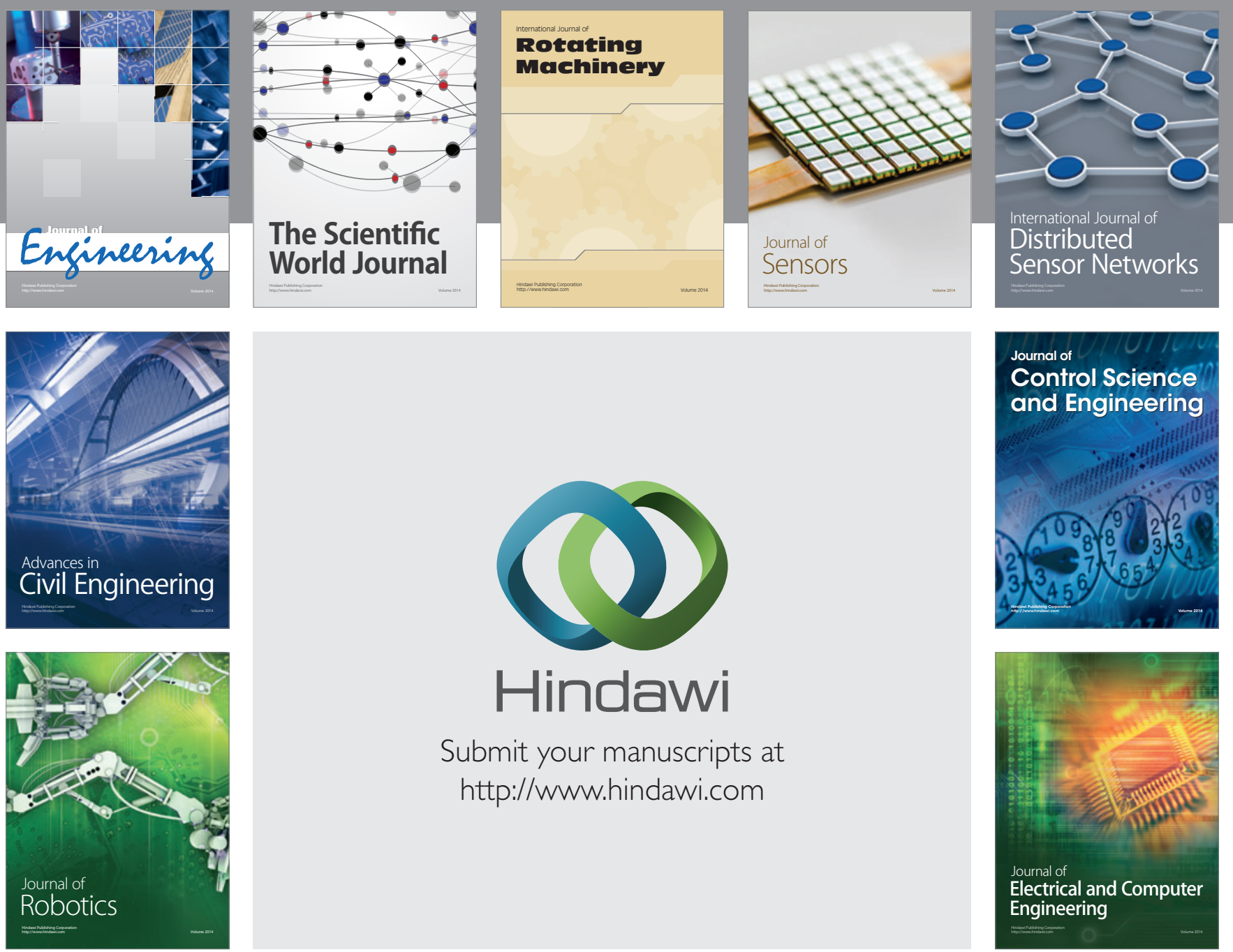

Submit your manuscripts at

http://www.hindawi.com
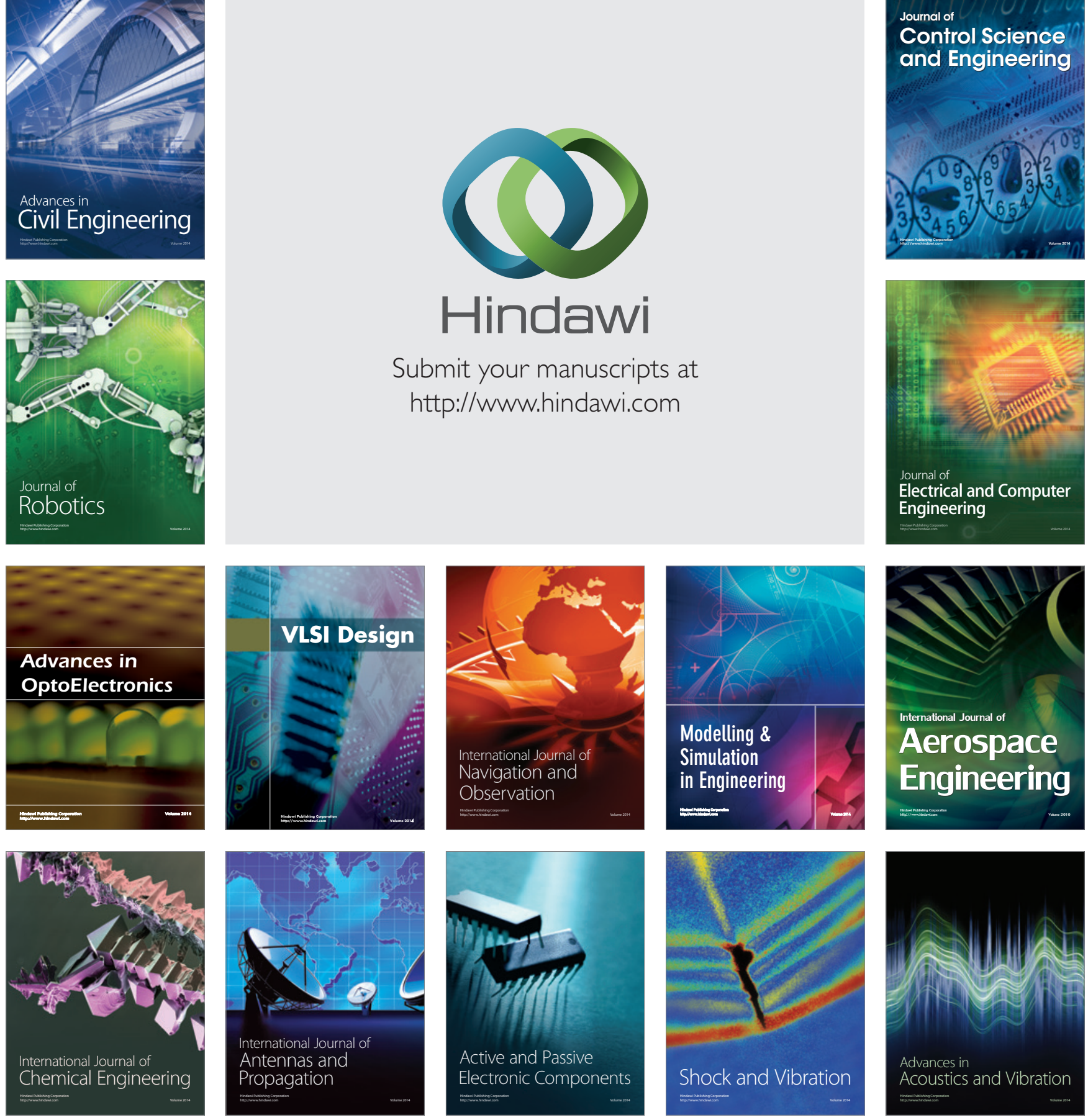